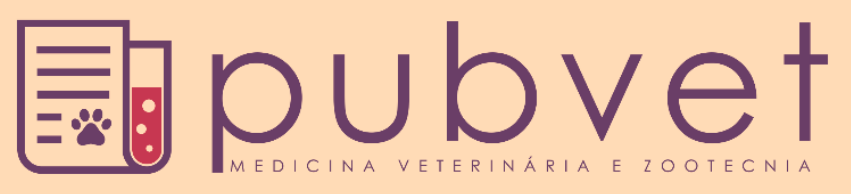

https://doi.org/10.31533/pubvet.v14n9a644.1-4

\title{
Lipoma gigante em região abdominal de cão: Relato de caso
}

\author{
Luciana Rangélia Malvina Souza de Castro ${ }^{1 *} \bullet$, Mariana Pacheco de Sousa ${ }^{1 \bullet}(\mathbb{D}$, Wanderson \\ Gabriel Gomes de Melo ${ }^{1} \odot$ (D), Artur Oliveira Rocha ${ }^{1} \bullet$, Catarina Rafaela Alves da Silva ${ }^{2}$, $^{\circ}$ \\ Thanisya Kelly de Paiva Brito ${ }^{3}{ }^{\circ}$, Francisco Lima Silva ${ }^{4}$
}

${ }^{I}$ Graduanda no curso de Bacharelado em Medicina Veterinária na Universidade federal do Piauí UFPI, Teresina - PI. Brasil.

${ }^{2}$ Médica veterinária, Doutora em ciência animal pela UFPI, Anestesista Hospital Veterinário Universitário-, Teresina - PI. Brasil. ${ }^{3}$ Médica veterinária autônoma Teresina - PI. Brasil.

${ }^{4}$ Docente do departamento de Clínica e Cirurgia Veterinária na Universidade Federal do Piauí. Teresina - PI. Brasil.

*Autor para correspondência. E-mail: luciana.rangelia@gmail.com

Resumo. O lipoma é uma neoplasia mesenquimal benigna de adipócitos, com maior frequência de apresentação nas formas subcutânea ou dérmica e sua ocorrência predominante está ligada à espécie canina. Geralmente se caracteriza por uma massa única, redonda, bem circunscrita, subcutânea, de crescimento lento, pode ser curado por excisão cirúrgica, sem necessidade de tratamento quimioterápico. E apesar da baixa periculosidade quanto a vida do animal, o bem-estar deste pode estar comprometido em virtude da sua característica de grande crescimento e formação de úlceras, com quadros de dor associados. Durante atendimento no Hospital Veterinário Universitário da Universidade Federal do Piaú, foi atendido um cão de 8 anos de idade, macho, sem raça definida, apresentando uma massa pendular na região abdominal, medindo aproximadamente $30 \mathrm{~cm}$ de comprimento, à palpação foi possível notar pequenas áreas nodulares ulceradas junto à grande massa. Para a confirmação do diagnóstico foi realizada citologia a partir de punção aspirativa por agulha fina à qual demonstrou presença de agrupamento de adipócitos. A ultrassonografia com Doppler colorido comprovou ausência de vascularização da massa e os exames radiográficos não apresentaram sinais de metástase. Procedeu-se o tratamento cirúrgico com extirpação área afetada. Foi instituído o tratamento pós-cirúrgico com Maxicam, Pentabiótico Veterinário, Cloridrato de Tramadol, limpeza local diária, aplicação de Rifocina e realização da bandagem compressiva. O tratamento foi eficaz animal teve excelente recuperação, sem sinais de reincidência.

Palavras chave: cães, gigante, pele, tumor

\section{Giant lipoma in dog abdominal region: Case report}

Abstract. Lipoma is a benign mesenchymal neoplasm of adipocytes, most commonly presented in subcutaneous or dermal forms and its predominant occurrence is linked to the canine species. It is usually characterized by a single, well-circumscribed, subcutaneous, slow-growing mass that can be cured by surgical excision without the need for chemotherapy. And despite the low danger to the life of the animal, the welfare of the animal may be compromised due to its characteristic growth and ulcer formation, with associated pain. During treatment at the University Veterinary Hospital of the Federal University of Piauí, was treated an 8-year-old male dog, with no defined breed, presenting a pendular mass in the abdominal region, measuring approximately $30 \mathrm{~cm}$ in length, on palpation was possible, note a small ulcerated nodular areas next to the large mass. To confirm the diagnosis, cytology was performed from fine needle aspiration, which showed the presence of adipocyte clustering. Color Doppler ultrasonography showed no mass vascularization and radiographic exams showed no signs of metastasis. 
Surgical treatment with extirpation of the affected area was performed. Post-surgical treatment with Maxicam, Veterinary Pentabiotic, Tramadol Hydrochloride, daily local cleaning, Rifocin application and compressive bandage were instituted. The treatment was effective the animal had excellent recovery with no signs of recurrence.

Keywords: dogs, giant, skin, tumor

\section{Lipoma gigante en la región abdominal del perro: Reporte de un caso}

Resumen. El lipoma es una neoplasia mesenquimatosa benigna de adipocitos, más comúnmente presentada en formas subcutáneas o dérmicas y su aparición predominante está relacionada con la especie canina. Por lo general, se caracteriza por una masa única, bien circunscrita, subcutánea, de crecimiento lento que puede curarse mediante escisión quirúrgica sin necesidad de quimioterapia. Y a pesar del bajo peligro para la vida del animal, el bienestar del animal puede verse comprometido debido a su crecimiento característico y formación de úlceras, con dolor asociado. Durante el tratamiento en el Hospital Veterinario Universitario de la Universidad Federal de Piauí, se atendió a un perro macho de 8 años, mestizo, con una masa pendular en la región abdominal, que midió aproximadamente $30 \mathrm{~cm}$ de longitud. Fui posible notar pequeñas áreas nodulares ulceradas al lado de la gran masa. Para confirmar el diagnóstico, la citología se realizó por aspiración con aguja fina, que mostró la presencia de agrupación de adipocitos. La ecografía Doppler a color no mostró vascularización masiva y los exámenes radiográficos no mostraron signos de metástasis. Se realizó tratamiento quirúrgico con extirpación de la zona afectada. Se instituyeron tratamientos posquirúrgicos con Maxicam, Pentabiótico Veterinario, Clorhidrato de Tramadol, limpieza local diaria, aplicación de Rifocin y vendaje compresivo. El tratamiento fue efectivo, el animal tuvo una excelente recuperación sin signos de recurrencia.

Palabras clave: perros, gigante, piel, tumor

\section{Introdução}

O lipoma é uma neoplasia mesenquimal benigna de adipócitos, com maior frequência de apresentação nas formas subcutânea ou dérmica. Tem ocorrência predominante ligada à espécie canina (Silva et al., 2017), apesar de ser encontrado relatos em grande parte das espécies domesticas. Geralmente se caracteriza por uma massa única, redonda, bem circunscrita, subcutânea, de crescimento lento, que pode ser curado, com facilidade, por excisão cirúrgica, sem necessidade de tratamento quimioterápico. Os locais de maior ocorrência de lipomas são tórax, abdome e membros (Fernandes et al., 2015).

Dentre os cães, o Labrador, Weimaraner, Dobermann, Dachshund, Cocker Spaniel e Poodle aparecem como os animais com maior número de casos da doença, que pode acometer qualquer raça. De modo geral, as fêmeas com idade próxima ou superior a 8 anos estão sob maior risco. Alguns pesquisadores sugerem a obesidade como um fator de predisposição. Contudo, deve-se levar em conta que, o acúmulo localizado de gordura pode ser confundido com lipomas.

Apesar de ser tido como um tumor benigno, de baixa periculosidade quanto a vida do animal, o bem-estar deste pode estar comprometido uma vez que o tumor tenha crescimento exagerado com comprometimento da qualidade de vida do animal. Em quadros mais graves, ulceras podem ser vistas, e consequentes quadros de dor associado (Bastos et al., 2017; Camargo et al., 2008; Gomes, 2015).

Objetivou-se neste trabalho relatar um caso de lipoma subcutâneo de $4 \mathrm{~kg}$, com dimensão de $14 \mathrm{~cm}$ x $28 \mathrm{~cm}$ x $38 \mathrm{~cm}$ na região abdominal de um cão Sem Raça Definida (SRD) atendido em Hospital Veterinário Universitário da cidade de Teresina-PI.

\section{Relato de caso}

Um canino macho de 4 anos, $32 \mathrm{~kg}$, SRD foi atendido no Hospital Veterinário Universitário de Teresina-PI, com uma massa pendular na região abdominal, medindo aproximadamente $30 \mathrm{~cm}$ de 
comprimento (Figura 1). A proprietária relatou que a massa aumentou progressivamente e que o animal não apresentava dor ao toque.

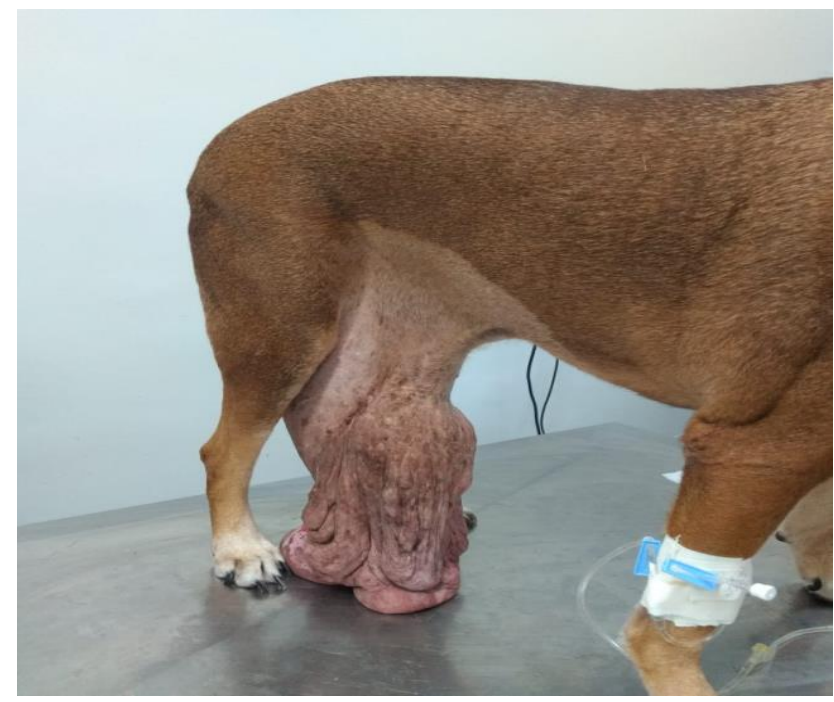

Figura 1. Massa pendular medindo $14 \mathrm{~cm}$ x $28 \mathrm{~cm}$ x $38 \mathrm{~cm}$, pesando $4 \mathrm{~kg}$, na região abdominal de cão SRD.

Ao exame físico do animal foram notadas temperatura retal, frequência cardíaca e frequência respiratória dentro dos parâmetros fisiológicos normais, ao se realizar a palpação foi possível notar pequenas áreas nodulares ulceradas junto à grande massa. Foram solicitados hemograma, bioquímicos, ultrassonografia, exames sorológicos e parasitológicos, radiografias e punção aspirativa por agulha fina (PAAF) (Figura 2).

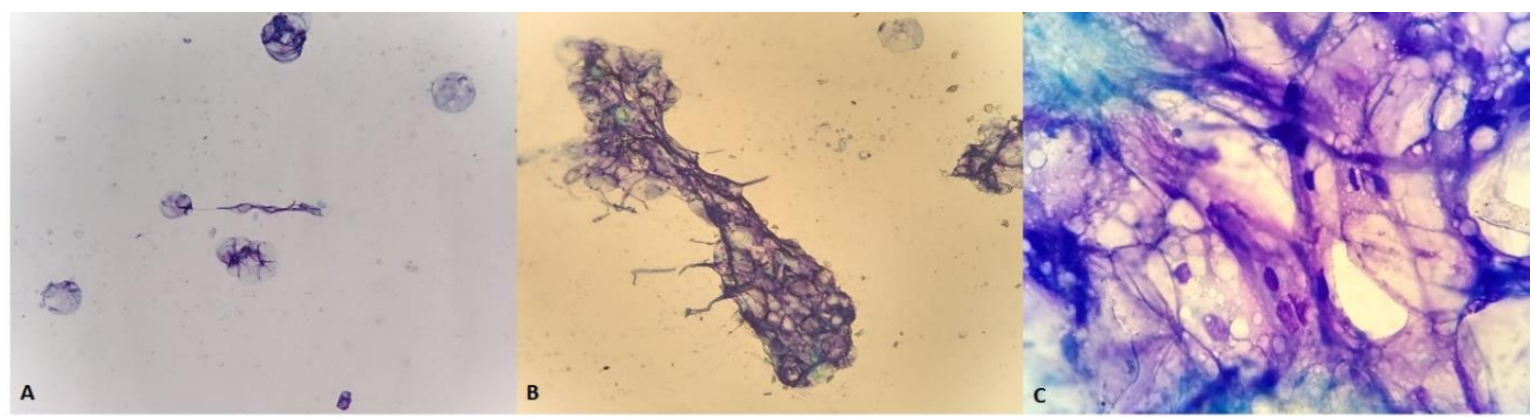

Figura 2. Citologia por aspiração por agulha fina. Agrupamento de adipócitos. Panótico. Ob. 10x (A e B), Ob. 100x (C).

Na ultrassonografia não foi possível observar vascularização para a massa no exame de Doppler colorido, e nem sinais de metástase nos exames radiográficos, o hemograma e os bioquímicos apresentaram valores dentro do padrão fisiológico. Feita a análise do histórico e dos resultados dos exames, foi sugerido o tratamento cirúrgico do animal.

O animal foi anestesiado utilizando acepram $0,05 \mathrm{mg} / \mathrm{kg}$ IM, morfina $0,5 \mathrm{mg} / \mathrm{kg} \mathrm{IM}$, propofol $4 \mathrm{mg} / \mathrm{kg} \mathrm{IV}$, intubação orotraqueal e manutenção com isoflurano vaporizado com $100 \%$ de O2, A massa foi incidida, sendo possível notar aspecto lipomatoso, na qual se iniciou a dissecção para a ressecção completa. Ao fim da dissecção, notou-se a presença de uma grande área de espaço morto, reduzida com utilização de fio mononáilon 2.0 e 3.0 e a colocação de drenos. Foi realizada uma bandagem compressiva na região abdominal com o objetivo de diminuir o máximo possível do espaço morto, com curativo trocados diariamente.

A terapia pós-cirúrgica foi feita com Maxicam 0,2\% IM SID, Pentabiótico Veterinário IM profundo a cada 4 dias, durante 16 dias, e Cloridrato de Tramadol 4mg/kg BID, limpeza local usando compressas de gases, Tergenvet, aplicação de Rifocina spray e realização da bandagem compressiva. $\mathrm{O}$ animal teve excelente recuperação e não apresentou sinais de reincidência. 


\section{Discussão}

Os lipomas são considerados neoplasias benignas que se originam dos adipócitos ou células gordurosas principalmente subcutâneas, por isso os animais mais predispostos a desenvolver esse problema são os animais obesos. Para a realização do diagnóstico foram realizados diversos exames, principalmente para descartar a possibilidade de neoplasia maligna (Miller et al., 1991; Paranhos, 2014).

Geralmente os lipomas afetam animais com média de 8 anos, porém no caso relatado o animal possuía apenas 4 anos, demonstrando a precocidade do desenvolvimento da massa, no entanto ele não costuma ser prejudicial ao metabolismo geral do animal, mas provoca um incomodo muito grande, principalmente aqueles que aumentarem muito de tamanho e peso (Silva et al., 2017; Viliotti et al., 2017).

$\mathrm{Na}$ cirurgia para a retirada do imenso nódulo, foram aplicadas técnicas, como uso de drenos e bandagens compressivas principalmente para diminuir a área de espaço morto no animal, pois esse é um dos maiores problemas na dissecção de lipomas muito robustos, que no animal apresentado possuía $4 \mathrm{~kg}, 38 \mathrm{~cm}$ de comprimento e $30 \mathrm{~cm}$ de largura (Fernandes et al., 2015).

No tratamento terapêutico de cirurgias muito cruentas é de suma importância fazer uma excelente analgesia, antibioticoterapia e anti-inflamatórios, para evitar sofrimento ao animal e principalmente infecções, que podem colocar a perder toda recuperação do animal (Bastos et al., 2017).

\section{Conclusão}

O lipoma é uma das neoplasias cutâneas de maior prevalência, e facilmente diagnosticada. O tratamento cirúrgico se faz suficiente não necessitando de quimioterapia. $\mathrm{O}$ tamanho é o principal entrave na terapia dos linfomas, dada a quantidade de espaço morto formado após a ressecção cirúrgica, dificultando a recuperação.

\section{Referências bibliográficas}

Bastos, R. S. C., Farias, K. M., Lopes, C. E. B., Pacheco, A. C. L., \& Araújo Viana, D. (2017). Estudo retrospectivo de neoplasias cutâneas em cães da região metropolitana de Fortaleza. Revista Brasileira de Higiene e Sanidade Animal, 11(1), 39-53.

Camargo, L. P., Conceição, L. G., \& Santos Costa, P. R. (2008). Neoplasias melanocíticas cutâneas em cães: estudo retrospectivo de 68 casos (1996-2004). Brazilian Journal of Veterinary Research and Animal Science, 45(2), 138-152. DOI: https://doi.org/10.11606/issn.16784456.bjvras.2008.26711

Fernandes, C. C., Medeiros, A. A., Magalhães, G. M., Szabó, M. P. J., Queiroz, R. P., Silva, M. V. A., \& Soares, N. P. (2015). Frequência de neoplasias cutâneas em cães atendidos no hospital veterinário da Universidade Federal de Uberlândia durante os anos 2000 a 2010. Bioscience Journal, 31(2), 541-548. DOI: https://doi.org/10.14393/bj-v31n2a2015-22371

Gomes, D. S. P. (2015). Neoplasias oculares do cão e gato: estudo retrospectivo de 5 anos: Vol. Master of. Universidade Lusófona de Humanidades e Tecnologias.

Miller, M. A., Nelson, S. L., Turk, J. R., Pace, L. W., Brown, T. P., Shaw, D. P., Fischer, J. R., \& Gosser, H. S. (1991). Cutaneous neoplasia in 340 cats. Veterinary Pathology, 28(5), 389-395. DOI: https://doi.org/10.1177/030098589102800506

Paranhos, C. A. (2014). Neoplasias cutâneas caninas: um estudo descritivo de 4 anos. In Medicina Veterinária: Vol. Master of. Universidade de Trás-os-Montes e Alto Douro.

Silva, F. L., Silva, T. S., Sousa, F. B., Sousa Junior, F. L., Pereira, L. J. C., Cruz Silva, J., \& Bezerra, F. B. (2017). Lipoma subcutâneo abrangendo as regiões cervical e peri-auricular de um canino: Relato de caso. PUBVET, 11(4), 363-370. DOI: https://doi.org/10.22256/pubvet.v11n4.363-370

Viliotti, T. A. A., Macedo, H. J. R., Chaves, M. G. L., Oliveira, R. C. R., Silva, H. F., \& Ferraz, R. E. O. (2017). Lipoma subcutâneo gigante em região lombo-sacral de um cão: Relato de caso. PUBVET, 12, 139. DOI: https://doi.org/10.22256/pubvet.v12n1a15.1-5 